Draft VERsion MARCH 21, 2021

Preprint typeset using $\mathrm{LAT}_{\mathrm{E}} \mathrm{X}$ style emulateapj v. 5/2/11

\title{
HI ABSORPTION FROM THE EPOCH OF REIONIZATION AND PRIMORDIAL MAGNETIC FIELDS
}

\author{
Evgenil O. VAsiliev ${ }^{1}$, Shiv K. Sethi ${ }^{2}$ \\ Draft version March 21, 2021
}

\begin{abstract}
We study the impact of primordial magnetic fields on the HI absorption from the Epoch of Reionization. The presence of these fields result in two distinct effects: (a) the heating of the haloes from the decay of magnetic fields owing to ambipolar diffusion, and (b) an increase in the number of haloes owing to additional matter fluctuations induced by magnetic fields. We analyse both these effects and show that the latter is potentially observable because the number of haloes along of line of sight can increase by many orders of magnitude. While this effect is not strongly dependent on the magnetic field strength in the range $0.3-0.6 \mathrm{nG}$, it is extremely sensitive to the magnetic field power spectral index for the near scale-free models. Therefore the detection of such absorption features could be a sensitive probe of the primordial magnetic field and its power spectrum. We discuss the detectability of these features with the ongoing and future radio interferometers. In particular, we show that LOFAR might be able to detect these absorption features at $z \simeq 10$ in less than 10 hrs of integration if the flux of the background source is $400 \mathrm{mJy}$.
\end{abstract}

\section{INTRODUCTION}

Future observations of the $21 \mathrm{~cm}$ line from neutral hydrogen from the redshift range $10-50$ will be an important source of information about, firstly, the study of global $21 \mathrm{~cm}$ signal after the recombination, which depends on the evolution of the thermal state of the universe (e.g. Pritchard \& Loeb 2010; Furlanetto et al. 2006; Sethi 2005; Gnedin \& Shaver 2004; Shaver et al. 1999), and, secondly, the formation of first stellar objects and reionization topology through the statistical properties of the $21 \mathrm{~cm}$ signal (e.g., Tozzi et al. 2000; Iliev et al. 2002). These observations can also help to put constraints on the relics of the inflation, such as super-heavy dark matter particles, which can produce ultra-high energy cosmic rays (Shchekinov \& Vasiliev 2004; Vasiliev \& Shchekinov 2006; Shchekinov \& Vasiliev 2007), annihilation and decays of dark matter particles (Furlanetto et al. 2006; Shchekinov \& Vasiliev 2007; Chuzhov 2008; Mvers \& Nusser 2008; Yuan et al. 2010; Cumberbatch et al. 2010; Natarajan \& Schwarz 2009) and primordial magnetic fields (Sethi \& Subramanian 2005; Tashiro \& Sugiyama 2006; Sethi \& Subramanian 2009).

The first two relics lead to extra ionization and heating of the gas both in the background (e.g., Shchekinov \& Vasiliev 2007; Chuzhov 2008; Mvers \& Nusser 2008) and in minihalos (Vasiliev \& Shchekinov 2013). The impact of primordial magnetic fields is more complicated. The decay of these fields in the post recombination era alters the thermal and ionization evolution of the gas which influences the properties of the $21 \mathrm{~cm}$ global signal (Sethi 2005; Sethi \& Subramanian 2005; Tashiro \& Sugivama 2006; [Schleicher et al. 2009). Also additional ionization and heating stimulate a growth of the molecular hydrogen fraction (Sethi et al. 2008), that can influence on the formation of first luminous objects.

More significant effect caused by primordial magnetic

\footnotetext{
${ }^{1}$ Southern Federal University, Rostov on Don 344090, Russia

${ }^{2}$ Raman Research Institute, Bangalore 560080, India
}

fields originate from the additional density fluctuations induced by these fields (Wasserman 1978; Kim et al. 1996; Subramanian et al. 1998; Gopal \& Sethi 2003). These effects act to both cut the number of haloes below the magnetic Jeans' scale and increase the number of haloes at larger scales owing to additional density perturbations (e.g. Sethi \& Subramanian 2005).

Unlike super-heavy dark particles and decaying dark matter, which have no observational counterpart in the local Universe, primordial magnetic field of nanoGauss strength may be the progenitor of the largescale magnetic fields observed in galaxies and clusters of galaxies with the coherence lengths up to $\simeq$ 10-100 kpc (for details of observations and theoretical models see e.g. Beck 2012; Widrow 2002). Even though these fields play an important role in various astrophysical processes, little is known about the origin of large-scale cosmic magnetic fields and their role in the evolutionary history of the universe. These fields could have originated from dynamo amplification of very small seed magnetic fields $\simeq 10^{-20} \mathrm{G}$ (e.g., Parker 1979; Zeldovich. Ruzmaikin \& Sokolov 1983; Brandenburg \& Subramanian 2005; Schober et al. 2013; Sur et al. 2012). It is also possible that much larger primordial magnetic fields $\left(\simeq 10^{-9} \mathrm{G}\right)$ were generated during the inflationary phase (Turner \& Widrow 1988; Ratra 1992; Widrow 2002; Ryu et al. 2012) and the large scale magnetic field observed at the present are the relics of these fields. In this paper, we investigate one possible implication of such primordial magnetic fields.

The strength of primordial magnetic field can be constrained by using a host of cosmological observables: the cosmic microwave background measurements, early reionization of the universe and HI signal from the era, cosmological gravitational lensing, and the study of Lyman- $\alpha$ clouds, etc. From the combination of WMAP and other CMBR data an upper limit $B_{0}<$ a few $\mathrm{nG}$ is obtained (Paoletti \& Finelli 2013; Yamazaki et al. 2010; Trivedi et al. 2012). CMBR observations are sensitive to large scale fields. For a sin- 
gle power law model, significantly tighter constraints are possible from the impact of these fields on smaller scales, which is also of direct relevance to us in this paper. From a host of constraints from the epoch of reionization, cosmological weak lensing, and the Lyman$\alpha$ data, one can obtain an upper limit on magnetic field strength in the range $\leq 0.4-1 \mathrm{nG}$ (Pandey \& Sethi 2013, 2012; Shaw \& Lewis 2012; Kahniashvili et al. 2010, 2013). Such fields can significantly modify the mass spectrum of the halos which results in potentially observable changes in the HI emission signal from the epoch of reionization (Kahniashvili et al. 2013; Sethi \& Subramanian 2009). In this paper, we study the influence of primordial magnetic fields (in the range $0.3-0.6 \mathrm{nG}$ ) on the statistical properties of the $21 \mathrm{~cm}$ absorption from the epoch of reionization. In principle, primordial magnetic fields can also be directly probed by polarization of the $21 \mathrm{~cm}$ background through Zeeman splitting (Cooray \& Furlanetto 2005).

We assume a $\Lambda$ CDM cosmology with the parameters $\left(\Omega_{0}, \Omega_{\Lambda}, \Omega_{m}, \Omega_{b}, h\right)=(1.0,0.76,0.24,0.041,0.73)$ (Planck Collaboration 2013; Spergel et al. 2007)

2. THE EFFECTS OF PRIMORDIAL TANGLED MAGNETIC FIELDS

We assume the primordial magnetic field to be statistically homogeneous, isotropic, and Gaussian. The twopoint function of the tangled fields (in comoving wave number) can be written as:

$$
\left\langle\tilde{B}_{i}(\mathbf{q}) \tilde{B}_{j}^{*}(\mathbf{k})\right\rangle=\delta_{D}^{3}(\mathbf{q}-\mathbf{k})\left(\delta_{i j}-k_{i} k_{j} / k^{2}\right) M(k)
$$

Here $M(k)$ is the magnetic field power spectrum and $k=|\mathbf{k}|$ is the comoving wavenumber. Here we assume: $M(k)=A k^{n}$ and $n$ is the magnetic field spectral index; we use $n \simeq-3$ throughout this paper (for justification of this range of spectral indices see e.g. (Pandey \& Sethi 2013) and references therein). The power law is cut-off at $k_{\max } \simeq 200\left(1 \mathrm{nG} / B_{0}\right)$ which is determined by the dissipation of magnetic fields in the pre-recombination era (for details see (Sethi \& Subramanian 2005) and references therein). Here we refer to magnetic field strength $B_{0}$ as the RMS $\left\langle\tilde{\mathbf{B}}^{2}(\mathbf{x})\right\rangle$ filtered at $k=1 \mathrm{Mpc}$.

The presence of magnetic field results in two distinct effects. The magnetic field dissipates in the collapsing halo owing to ambipolar diffusion which heats the halo. The spatial part of the heating rate by ambipolar diffusion can be computed (for details see (Sethi \& Subramanian 2005)):

$$
\left.\left\langle\left(\nabla_{\mathbf{x}} \times \tilde{\mathbf{B}}\right) \times \tilde{\mathbf{B}}\right)^{2}\right\rangle=\frac{7}{3} \int d k_{1} \int d k_{2} M\left(k_{1}\right) M\left(k_{2}\right) k_{1}^{2} k_{2}^{4}
$$

The presence of primordial magnetic fields also result in additional matter perturbations at small scales. The magnetic-field induced matter power spectrum $P(k) \propto$ $k^{3 n+7}$ for $n \leq-1.5$ and it is sharply cut-off at magnetic Jeans' scale $k_{J} \simeq 15\left(1 \mathrm{nG} / B_{0}\right)$ (Kim et al. 1996; Gopal \& Sethi 2003). This additional power at small scales changes the number of haloes at masses of interest $10^{6} \mathrm{M}_{\odot} \leq M \leq 10^{8} \mathrm{M}_{\odot}$. If the haloes in this mass range fail to form stars then they would be observable in $\mathrm{HI}$ absorption.

In this paper we take into account both these effects: (a) additional heating due to ambipolar diffusion, and (b) the impact of magnetic-field induced small scale power on matter power spectrum.

\section{THE MODEL OF COLLAPSING HALO, HI OPTICAL} DEPTH, AND THE NUMBER OF HALOES

\subsection{Dark matter, gas dynamics and magnetic fields}

To model the evolution of the dark matter we follow the prescription given by Ripamonti (2007). The dark matter mass, $M_{D M}=\Omega_{D M} M_{h a l o} / \Omega_{M}$, is assumed to be enclosed within a certain truncation radius $R_{t r}$, inside which the dark matter profile is a truncated isothermal sphere with a flat core of radius $R_{\text {core }}$. The parameter $\xi=R_{\text {core }} / R_{\text {vir }}$ is taken to be 0.1 for all simulations. Such a description is used to mimic the evolution of a simple top-hat fluctuation (e.g. Padmanabhan 1993). The resulting dark matter profile has a flattened form (Burkert 1995).

Dynamics of baryons is described by a 1D Lagrangian scheme similar to that proposed by Thoul \& Weinberg (1995); a reasonable convergence is found at a resolution of 1000 zones over the computational domain. Chemical and ionization composition includes a standard set of species: $\mathrm{H}, \mathrm{H}^{+}, \mathrm{H}^{-}, \mathrm{He}, \mathrm{He}^{+}, \mathrm{He}^{++}, \mathrm{H}_{2}, \mathrm{H}_{2}^{+}$, $\mathrm{D}, \mathrm{D}^{+}, \mathrm{D}^{-}, \mathrm{HD}, \mathrm{HD}^{+}$and $e$, with the corresponding reaction rates (Galli \& Palla 1998; Stancil et al. 1998). The energy equation includes heating owing to ambipolar diffusion and different radiative losses in the primordial plasma: Compton cooling, recombination and bremsstrahlung radiation, collisional excitation of HI (Cen 1992), $\mathrm{H}_{2}$ (Galli \& Palla 1998) and HD (Flower 2000; Lipovka et al. 2005). Our computation starts at redshift $z=100$. The initial parameters - gas temperature, chemical composition and other quantities - are taken from one-zone calculations that begin at $z=1000$ with values at the end of recombination: $T_{\text {gas }}=T_{C M B}, x[\mathrm{H}]=0.9328, x\left[\mathrm{H}^{+}\right]=0.0672, x[\mathrm{D}]=$ $2.3 \times 10^{-5}, x\left[\mathrm{D}^{+}\right]=1.68 \times 10^{-6}$ (see references and details in Ripamonti 2007, Table 2).

In the early evolution of the halo, when it is still in the expansion phase and the overdensity is very small, the magnetic field strength scales as $(1+z)^{2}$. The initial condition is set during this phase. During the collapse phase of the halo, we assume the magnetic field strength to scale as its flux-frozen evolution with the gas density $\rho$ : $B_{0} \propto \rho^{2 / 3}$ (e.g. Sethi et al. 2008; Schleicher et al. 2009; Sethi et al. 2010). It should be noted that in both the expansion and the collapse phase, magnetic field scales as $\rho^{2 / 3}$ with the gas density.

The heating rate due to the ambipolar diffusion is computed from Eq. (2); during the expansion phase this rate can be written as (Schleicher et al. 2008):

$$
L_{A}=\frac{B^{4}}{l_{a}^{2}} \frac{x_{H I}}{16 \pi^{2} \gamma x_{e} \rho^{2}}
$$

where $B=B_{0}(1+z)^{2}, \gamma=3 \times 10^{-9} /\left(2 m_{p}\right)$ and

$$
l_{a}=1.32 \times 10^{22} \frac{B_{0}}{10^{-9}} \frac{A}{1+z}
$$

$A$ depends on the cosmological parameters $\Omega_{m}, h, \Omega_{b}$ and the magnetic field power spectrum $M(k)$. Eq. (3) can be extended to the collapse phase using the flux-frozen condition discussed above. 




FIG. 1.- The density, temperature, velocity and molecular hydrogen profiles across the collapsing halo, for different values of magnetic field strengths. The two panel correspond to $M=10^{7}, 10^{8} \mathrm{M}_{\odot}$, respectively for virialization redshift $z_{\mathrm{vir}}=10$.

Using the method and initial conditions described above we follow the evolution of minihaloes with masses in the range $M=10^{6}-10^{9} \mathrm{M}_{\odot}$; the virialization redshift of these haloes is in the redshift range $z=10-15$. In Figure 1 we show the density, temperature, velocity, and molecular hydrogen profiles for two haloes, including the effects of magnetic fields. We present the radial profiles of haloes with $M=10^{7}, 10^{8} \mathrm{M}_{\odot}$ virialized at $z_{\text {vir }}=10$ for standard recombination $(B=0)$ and several values of initial magnetic strength: $B=0.1,0.3,0.6$ and $1 \mathrm{nG}$. The most notable feature with direct impact on results presented in this paper is the change in the temperature profile. As seen in the Figure, the presence of magnetic fields results in an increase in temperature across the halo, which has a direct bearing on the strength of the observed HI profile.

\section{2. $21 \mathrm{~cm}$ optical depth and equivalent width}

The spin temperature of the HI $21 \mathrm{~cm}$ line is determined by atomic collisions and the scattering of ultraviolet (UV) photons (Field 1958; Wouthuysen 1952). In our calculations we used collisional coefficients from Kuhlen et al. (2006) and Liszt (2001). Magnetic fields provide the extra heating source. We do not include
Lyman- $\alpha$ pumping in our study. We note that the inclusion of Lyman- $\alpha$ scattering serves to couple the matter temperature with the spin temperature and plays a crucial role in the observability of IGM HI in emission from the epoch of reionization (e.g. Sethi 2005; Gnedin \& Shaver 2004). However, we consider only absorption from collapsing haloes in the redshift range $10<z<15$ against bright radio sources. In such haloes, the spin temperature is coupled to the matter temperature through collisions and Lyman- $\alpha$ doesn't play an important role.

The optical depth along a line of sight at a frequency $\nu$ is:

$$
\begin{array}{r}
\tau_{\nu}=\frac{3 h_{p} c^{3} A_{10}}{32 \pi k \nu_{0}^{2}} \int_{-\infty}^{\infty} d x \frac{n_{\mathrm{HI}}(r)}{\sqrt{\pi} b^{2}(r) T_{s}(r)} \\
\times \exp \left[-\frac{\left[v(\nu)-v_{l}(r)\right]^{2}}{b^{2}(r)}\right]
\end{array}
$$

where $r^{2}=\left(\alpha r_{\text {vir }}\right)^{2}+x^{2}, \alpha=r_{\perp} / r_{\text {vir }}$ is the dimensionless impact parameter, $v(\nu)=c\left(\nu-\nu_{0}\right) / \nu_{0}, v_{l}(r)$ is the infall velocity projected along the line of sight, $b^{2}=2 k T_{k}(r) / m_{p}$ is the Doppler parameter.

The observed line equivalent width: $W_{\nu}^{o b s}=W_{\nu} /(1+$ 


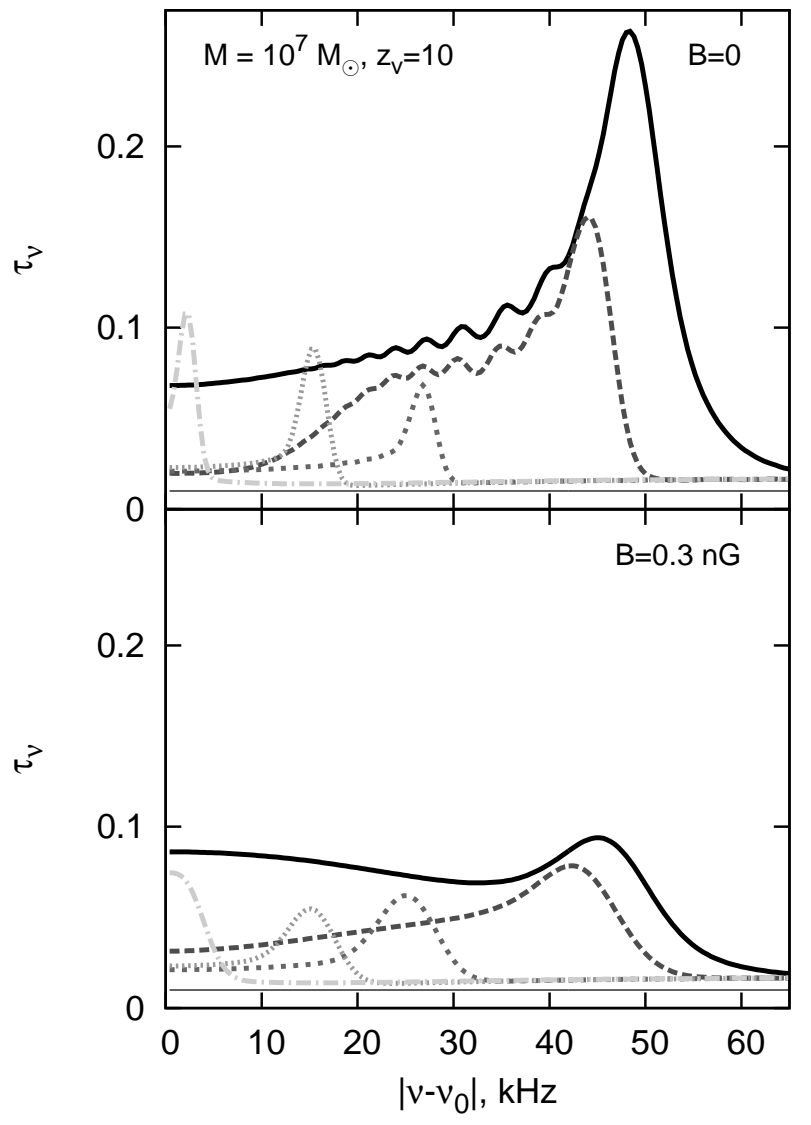

FIG. 2.- Optical depth at the impact parameters $0.1,0.3,1,1.5,3 r / r_{\text {vir }}$ (solid, dash, dot, short dash and dotdash lines, correspondingly) for a halo $M=10^{7} M_{\odot}$ virialized at $z_{\mathrm{vir}}=10$ in the standard recombination model $(B=0)$ and in the presence of primordial magnetic fields with $B=0.3 \mathrm{nG}$. The $\mathrm{x}$-axis denotes the frequency width of the signal in the rest frame of the halo.

$z)$, where the intrinsic equivalent width is

$$
W_{\nu}=\int_{\nu_{0}}^{\infty}\left(1-e^{-\tau_{\nu}}\right) d \nu-\int_{\nu_{0}}^{\infty}\left(1-e^{-\tau_{\mathrm{IGM}}}\right) d \nu
$$

where $\tau_{\text {IGM }}$ is the optical depth of the background neutral IGM. Throughout this paper, we assume $\tau_{\text {IGM }}=0$

In Figure 2 we show the expected absorption profiles in the presence of magnetic fields. Additional heating owing to ambipolar diffusion acts to lower the expected optical depth by up to a factor of $2-3$ depending on the strength of the field and the impact factor.

\subsection{Number of haloes}

We use the Press-Schechter formalism to compute the number of haloes in a given mass range at a redshift. This allows us to compute the number of haloes that intersect a given line of sight in a redshift range $z$ and $z+d z$ :

$$
N(z)=\int d M\left(\frac{d n}{d M}\right) \pi r_{\perp}^{2} \frac{H_{0}^{-1} d z}{\Omega^{1 / 2}(1+z)^{5 / 2}}
$$

Here $r_{\perp}$ is the impact factor and for our computation we take the maximum impact factor $r_{\perp}=3 r_{\text {vir }}$ for any mass; $d n / d M$ is the mass function of haloes (in $M p c^{-3} M_{\odot}^{-1}$ ).

The main impact of primordial magnetic fields is on the number of haloes in the mass range $10^{6} \mathrm{M}_{\odot} \leq M \leq$

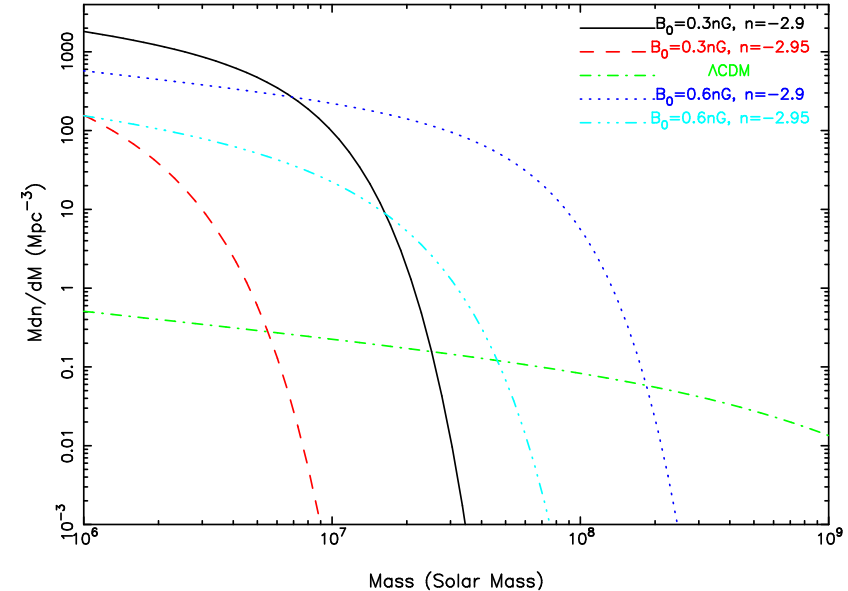

Fig. 3.- Comoving mass function is shown for different models at $z=10: \Lambda \mathrm{CDM}$ model (dot-dashed curve), solid and dotted line ( $B_{0}=0.3 \mathrm{nG}, n=-2.9,-2.95$, respectively), dashed and dot-dotdot-dashed line ( $B_{0}=0.6 \mathrm{nG}, n=-2.9,-2.95$, respectively).

$10^{8} \mathrm{M}_{\odot}$. Figure 3 shows the mass function of haloes for the models with and without the primordial magnetic fields. The number of haloes in the mass range of interest could be orders of magnitude larger than in the usual $\Lambda$ CDM model. This is owing to the extra power at small scales induced by primordial magnetic fields. This extra power increases the mass dispersion $\sigma(M, z)$ at mass scales comparable to the magnetic Jeans' mass; in particular such an increase might result in 1- $\sigma$ collapse of such haloes while the $\Lambda$ CDM model only allows nearly $3-\sigma$ overdensities to collapse at $z=10$ (e.g. Sethi \& Subramanian 2005). In the Press-Schechter formulation, the mass function of haloes: $d n / d M \propto \exp \left[-\delta_{c}^{2} /\left(2 \sigma^{2}(M, z)\right)\right]$ with $\delta_{c}=1.68$; this results in a sharp increase in the number of haloes at small scales. Two other notable features of the Figure are: (a) an increase in the strength of magnetic field results in a decrease in the number of haloes at small mass scales and an increase for larger masses, this feature is caused by the increase of magnetic Jeans' length with magnetic field strength, and (b) the number of haloes is seen to be extremely sensitive to the magnetic field spectral index. For $n \simeq-3$, the mass dispersion, $\sigma(M) \propto(n+3)$. As the number of haloes is exponentially sensitive to the value of mass dispersion, the number of haloes sharply decreases as $n$ approaches -3 .

For $\Delta z=0.2$ (corresponding to a frequency width $\Delta \nu \simeq 2.4 \mathrm{MHz}$ at $z \simeq 10$ ) and the $\Lambda \mathrm{CDM}$ model, $N(z) \simeq 0.1$ or one out of ten lines of sight is likely to intersect such a halo. For the magnetic field of strength $B_{0}=0.3 \mathrm{nG}$ and $n=-2.9$, we get $N(z) \simeq 18$.

To underline the impact of primordial magnetic fields, we define an effective optical depth:

$$
\begin{aligned}
& \tau_{\text {eff }}(z)=\int d r_{\perp} \int d M\left(\frac{d n}{d M}\right)(z) \\
& \times 2 \pi r_{\perp} \frac{H_{0}^{-1} d z}{\Omega^{1 / 2}(1+z)^{5 / 2}} \tau\left(M, r_{\perp}, z\right)
\end{aligned}
$$

$\tau_{\text {eff }}(z)$ is weighted by $\tau\left(M, r_{\perp}, z\right)$ and scales as the number of haloes so it captures both the decrease in optical depth owing to additional heating and the increase of number of haloes in the presence of magnetic field. 


\section{RESULTS}

To illustrate the difference between the $\Lambda$ CDM model and the effects of primordial magnetic fields, we simulate absorption spectra in the redshifted HI line.

Eq. (6) gives the number of haloes in a redshift interval (or equivalently for a given frequency channel width). For simulating the absorption spectrum we choose the redshift bin $d z$ to be such that $N(z) \ll 1$. For each channel, a Poisson number is drawn with the average given by $N(z)$. Since $N(z) \ll 1$, this number is either 0 or 1 . To determine the mass and impact factor of the intervening halo, random numbers are drawn from the mass function for the respective cases and probability density for the impact factor. Given the optical depth as function of mass, impact factor, and the frequency, this procedure allows us to simulate synthetic spectra.

In Figure 4 we show the simulated spectra for two cases: $B_{0}=0.3 \mathrm{nG}$ and $B_{0}=0.6 \mathrm{nG}$ for $n=-2.9$ and $n=-2.95$ at $z=10$. The channel width in the spectra is $\simeq 1 \mathrm{kHz}$ and the total frequency coverage is $\simeq 20 \mathrm{MHz}$.

As seen in the Figure, the number of intersecting haloes in the two cases are comparable, even though the number density of haloes is larger in the former case (Figure 3). This is because the number of intersecting haloes scales as $M^{1.66} d n / d m$ (Eq 6), which puts higher weights on haloes of larger mass as compared to the distribution shown in Figure 3. Also this combination picks a maximum at certain mass scales: for $B_{0}=0.3,0.6 \mathrm{nG},(n=$ $-2.90)$ the mass scales are $7 \times 10^{5} \mathrm{M}_{\odot}$ and $6 \times 10^{6} \mathrm{M}_{\odot}$, respectively (for $\Lambda \mathrm{CDM}$ model the equivalent mass is $\left.2 \times 10^{8} \mathrm{M}_{\odot}\right)$. As the Figure shows, the spectrum is not particularly sensitive to the strength of magnetic field, for a given value of the spectral $n$, in this range of magnetic field strengths.

However, the number of haloes along a line of sight is very sensitive to the spectral index $n$. For $n=-2.95$, the number of such sources falls by more than an order of magnitude for both magnetic field strengths shown in the Figure. This means that the observed spectra could be a strong discriminator of the magnetic field power spectral index.

In Figure 5 we show the simulated spectra at $z=15$. The sharp fall in the number of absorption features is owing to the decrease in mass dispersion at higher redshifts.

As noted above, the $\Lambda \mathrm{CDM}$ model predicts roughly one intersecting halo for the frequency coverage displayed in Figure 4. Therefore, in principle, if deep absorption features seen in Figure 4 are detected towards a few lines of sight, it could be an indication of a process which produces extra matter fluctuations at small scales. In particular, the presence of a large number of sources along a line of sight (Figure 4) could be used to extract information about the magnetic field parameters.

\subsection{Detectability of $H I$ absorption}

As discussed above, the main impact of the inclusion of magnetic field in our analysis is a sharp increase in the number of absorption lines (Figure 4). Each of the absorption feature has a width of $\simeq 10 \mathrm{kHz}$ (in the observer frame for $z \simeq 10$, Figure 2).

This leads to at least three distinct possibilities of the detection of the signal:

(a) owing to a large number of absorption features, the probability of deep, narrow features that arise when the impact parameter is small increases (Figure 2). Figure 4 shows that many such features have optical depths in the excess of $\tau=0.05$; such features are detectable at frequency resolutions $\simeq 1 \mathrm{kHz}$,

(b) at the frequency resolution comparable to the line width we expect significant signal. Also there is a reasonable probability of line blending owing to high density of such features,

(c) at even lower frequency resolution, one can hope to detect the combined effect of many lines blending into a broad feature (Xu et al. 2011).

Ongoing experiments such as Murchison Widefield Array (MWA) and Low-Frequency Array (LOFAR) can in principle detect the absorption signal from the redshifted HI line. MWA can achieve channel widths $\simeq 40 \mathrm{kHz}$ and total instantaneous bandwidth of $32 \mathrm{MHz}$ in the frequency range of interest $(80-150 \mathrm{MHz} 3$. LOFAR can have a channel width of $\leq 1 \mathrm{kHz}$ with total band width of roughly $75 \mathrm{MH} 4^{4}$. LOFAR spectral resolution is comparable to the spectra shown in Figure 4.

Given the angular resolution of these experiments, one of the challenges in the detection of these absorption features from the epoch of reionization is to distinguish high redshift bright continuum sources from low redshift sources. MWA has an angular resolution of nearly 4' and a confusion noise $\simeq 10 \mathrm{mJy}$. On the other hand, LOFAR presents a better prospect with an angular resolution $\simeq 10^{\prime \prime}$ which gives a confusion noise $\simeq 100 \mu \mathrm{Jy}$. LOFAR can reach a sensitivity of $200 \mu \mathrm{Jy}$ in 8 hours of integration for a bandwidth of $3.66 \mathrm{MHz}$ in the frequency range $100-200 \mathrm{MHz}$. This corresponds to a line sensitivity (for a channel width $\Delta \nu \simeq 1 \mathrm{kHz}$ ) of nearly $14 \mathrm{mJy}$. To detect a line feature with an optical depth $\simeq 0.05$ (Figure 4) at $5 \sigma$ level, a source of a few Jansky flux density would be needed. Significantly longer integration time would be needed for fainter sources.

To investigate the possibility of detecting HI features at lower frequency resolution, we show equivalent widths, $W_{\nu}$ (Eq. 4), for spectra at two different frequency resolutions in Figure 6 and 7 . Even though the equivalent widths are clustered around $W_{\nu} \leq 0.2 \mathrm{kHz}$ in Figure 6 , there are a number of features at higher equivalent widths, e.g. the lower, left panel of Figure 6 has two features in the range $W_{\nu}=0.55-0.6 \mathrm{kHz}$, that arise from blending of two lines. If the threshold of detection is $\tau=0.02$, then these features can be detected with $\Delta \nu \simeq 30 \mathrm{kHz}$. Or at such spectral resolutions, one obtains a gain in the signal-to-noise of a factor of two over detecting a feature of $\tau=0.05$ with $\Delta \nu \simeq 1 \mathrm{kHz}$.

In Figure 7, we display equivalent widths for spectra smoothed at $\Delta \nu \simeq 100 \mathrm{kHz}$. This allows us to assess the feasibility of detecting $\mathrm{HI}$ absorption features at low resolutions. The distribution of equivalent widths

\footnotetext{
3 http://www.mwatelescope.org/index.php/telescope

4 https://www.astron.nl/radio-observatory/astronomers/ users/technical-information/frequency-selection/ station-clocks-and-rcu
} 


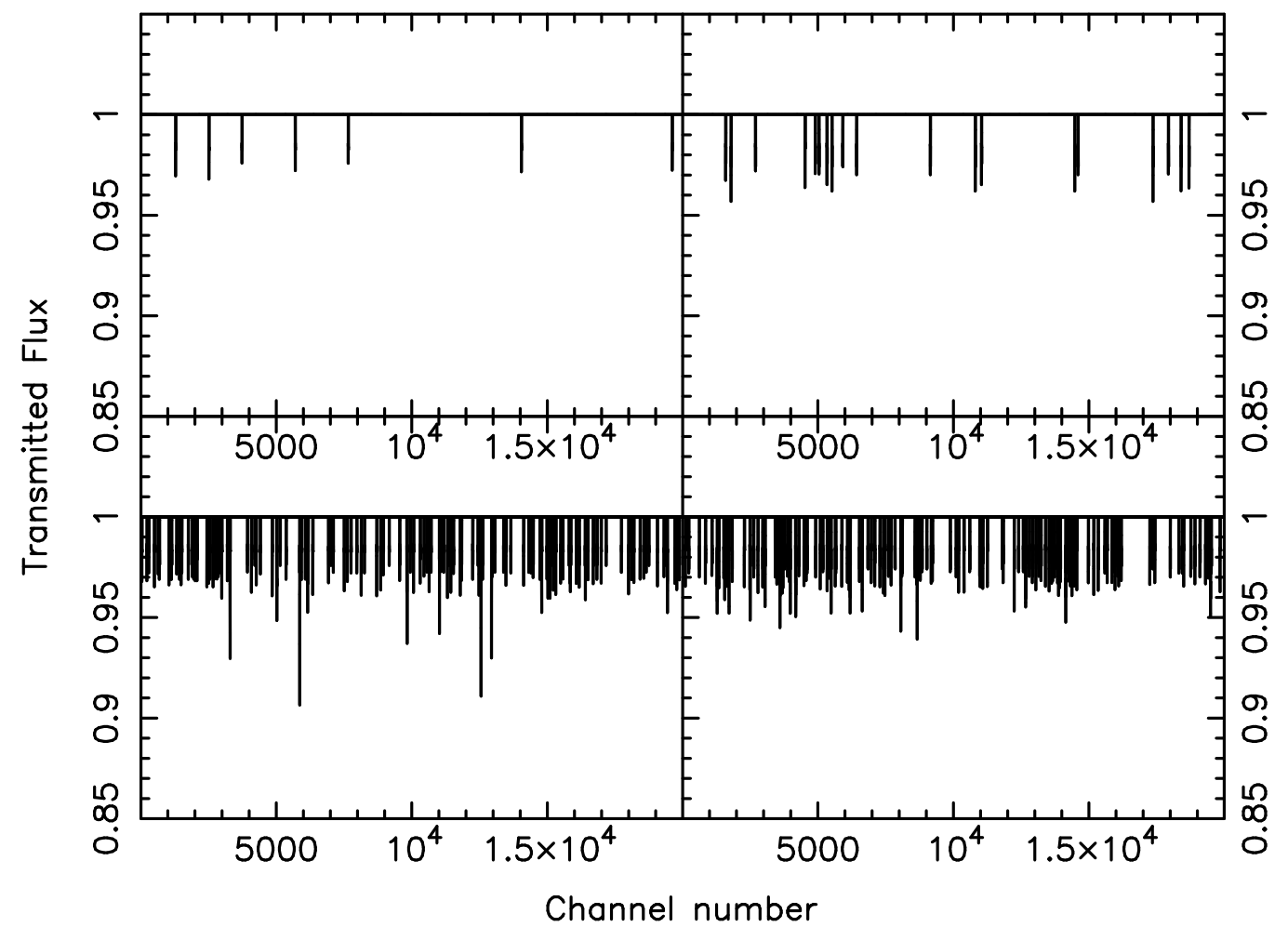

FIG. 4. - Simulated spectra are displayed for two values of magnetic field $B_{0}=0.3,0.6 \mathrm{nG}$ (left and right panels, respectively) and for $n=-2.9,2.95$ (lower and upper panels, respectively) at $z=10$. The spectra have a channel width of $1 \mathrm{kHz}$. The total band width of each spectrum is $\simeq 20 \mathrm{MHz}$. Lower panels: There are 141 (7) intersecting haloes in the left lower (upper) panels and 147 (19) in the right lower (upper) panels.

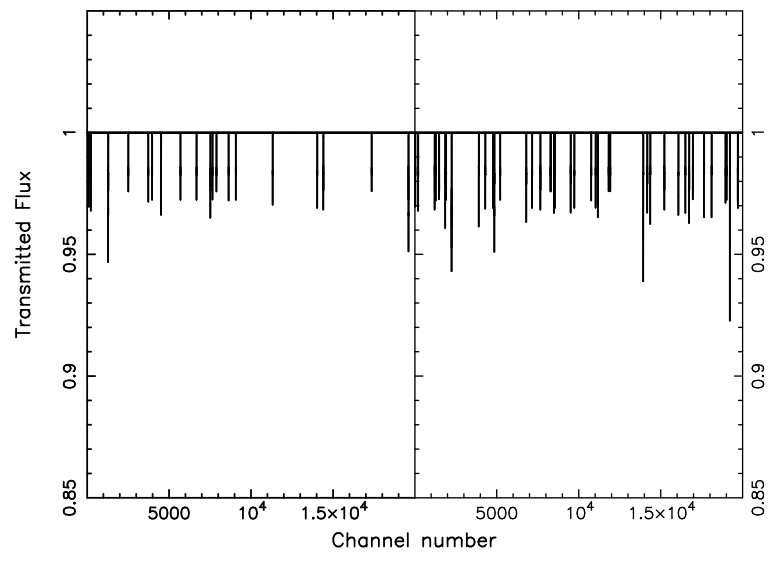

Fig. 5.- Simulated spectra are displayed for two values of $B_{0}=$ $0.3,0.6 \mathrm{nG}$ (left panel) and for $n=-2.9$ (right panel) at $z=15$. The spectra have a channel width of $1 \mathrm{kHz}$. The total band width of the spectra displayed is $\simeq 20 \mathrm{MHz}$. The number of intersecting haloes in the left (right) panels are 26 (45).

is seen to shift to larger values for the smoothed spectrum. This is owing to blending of densely packed spectral lines as the resolution is decreased. The most notable feature of the figure is that many features are seen to have equivalent widths $\simeq 2 \mathrm{kHz}$, nearly three times the largest values for the high resolution spectra. This means that the decline in peak optical depth as the spectra are smoothed is compensated by the inclusion of more sources. In other words, if the threshold optical depth of detection is 0.02 , it can be detected even with a resolution of $\Delta \nu \simeq 100 \mathrm{kHz}$. More specifically, using the parameters of LOFAR, such features are detectable by LOFAR at $5-\sigma$ level for a source flux density of $\simeq 400 \mathrm{mJy}$ in 8

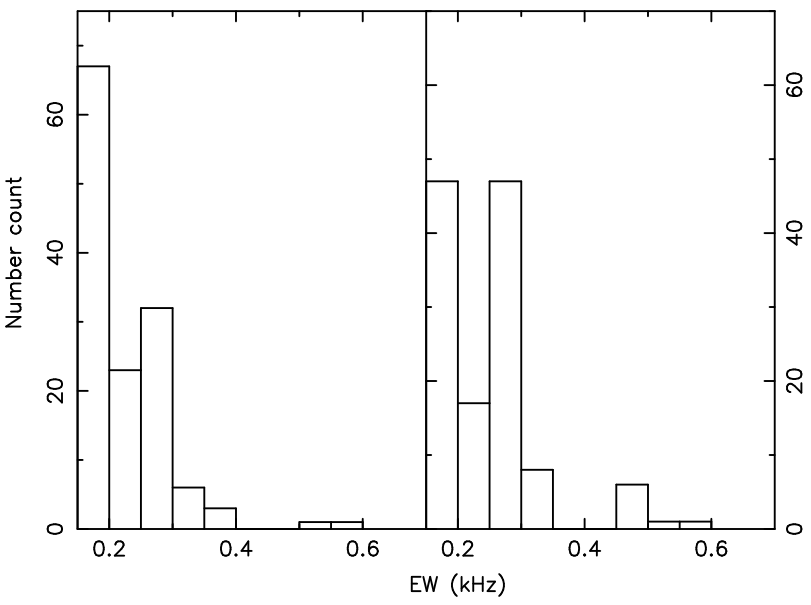

FIG. 6.- Histograms of equivalent width distribution for the spectra in Figure 4 (lower panels),

hours of integration.

As noted above, a few such sources might suffice to reveal the nature of magnetic field-induced extra matter power. How likely are such bright radio sources at $z \simeq$ 10 ? The radio source J0924-2201 has $z \simeq 5.2$ with a flux $F_{\nu}=0.55 \mathrm{Jy}$ at $230 \mathrm{MHz}$ (Carilli et al. 2007). Such a steep spectrum source might be brighter at $\nu \simeq 130 \mathrm{MHz}$ (redshifted HI frequency at $z \simeq 10$ ) with flux comparable to its value at $235 \mathrm{MHz}$. Such rare radio sources might provide a suitable setting for understanding the nature of density perturbations during the EoR.

However, the expected radio flux distribution of sources from the epoch of reionization is highly uncertain and for for fainter sources the integration time 


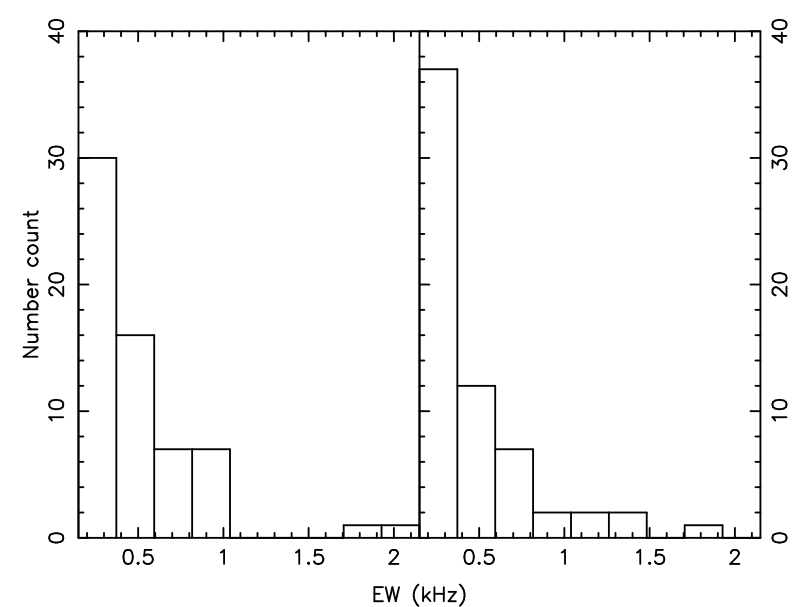

Fig. 7.- Histogram of equivalent width distribution for the spectra in Figure 4 (lower panels), smoothed by a spectral width of $100 \mathrm{kHz}$.

TABLE 1

THE NUMBER OF INTERSECTING HALOES PER UNIT REDSHIFT AND THE EFFECTIVE OPTICAL DEPTH (EQ. 8) PER UNIT REDSHIFT ARE LISTED FOR A RANGE OF MODELS WITH AND WITHOUT PRIMORDIAL MAGNETIC FIELDS.

\begin{tabular}{ccc}
\hline \hline & $\mathrm{dN}(\mathrm{z}) / \mathrm{dz}$ & $d \tau_{\text {eff }} / d z$ \\
\hline$\Lambda \mathrm{CDM}, z=10$ & 0.25 & 0.38 \\
$B_{0}=0.3 \mathrm{nG}, n=-2.9, z=10$ & 92 & 198 \\
$B_{0}=0.3 \mathrm{nG}, n=-2.95, z=10$ & 2 & 3.4 \\
$B_{0}=0.6 \mathrm{nG}, n=-2.9, z=10$ & 95 & 210 \\
$B_{0}=0.6 \mathrm{nG}, n=-2.95, z=10$ & 10 & 22 \\
$B_{0}=0.3 \mathrm{nG}, n=-2.9, z=15$ & 12 & 35 \\
$B_{0}=0.6 \mathrm{nG}, n=-2.9, z=15$ & 18 & 69 \\
& & \\
\hline
\end{tabular}

might become unrealistically large, e.g. for a $40 \mathrm{mJy}$ source, $800 \mathrm{hrs}$ of integration time would be needed. The planned radio interferometer SKA will be ideal for detecting these features: its sensitivity is projected to reach $400 \mu \mathrm{Jy}$ in one minute integration in the frequency range 70-300 MHz with sub-arcsec resolution, which will suppress the impact of confusion nois 5 .

In this paper we only considered absorption from collapsed haloes which are rarer but give rise to large optical depths. In addition, we could have mildly overdense regions (with density contrasts $\delta \simeq 1-10$ ) in the neutral gas (Mack \& Wyithe 2012). These regions give rise to smaller optical depths but their effect might be detectable through statistical methods, based on distribution of radio sources during the epoch of reionization (Mack \& Wyithe 2012). Magnetic fields alter the density field at all scales and therefore would also change the statistical properties of such regions (e.g., Pandey \& Sethi 2013). We hope to return to this issue in a later work.

In Table 1, we list $\tau_{\text {eff }}$ for a range of models. As noted above, this measure captures the impact of both magnetic field heating and the change in the number of haloes.

\subsection{Comparison between magnetic heating, dark matter decay and $X$-ray heating}

\footnotetext{
${ }^{5}$ https://www.skatelescope.org/
}

The evolution of the observed number and optical depths of absorption features is sensitive to the evolution of heating mechanisms of the IGM. Here we briefly compare the relative impact of X-ray heating, dark matter decay models, and the magnetic field heating.

Using Eq. (3) the heating rate due to ambipolar diffusion can be written as $\left[\mathrm{K} \mathrm{s}^{-1}\right]$ :

$$
\frac{2 L_{A}}{3 k n H(z)} \simeq 1.2 \times 10^{4}\left(\frac{B_{0}}{10^{-9}}\right)^{2}\left(\frac{1+z}{10}\right)^{-1 / 2}\left(\frac{10^{-4}}{x_{e}}\right)
$$

One can compare this rate to the heating rates due to $\mathrm{X}$-ray and decaying dark matter. The total normalized X-ray emissivity $\epsilon_{X}$ can be written as (Furlanetto 2006)

$$
\frac{2 \epsilon_{X}}{3 k n H(z)}=5 \times 10^{4} f_{X}\left[\frac{f_{*}}{0.1} \frac{d f_{\text {coll }} / d z}{0.01} \frac{1+z}{10}\right],
$$

here $d f_{\text {coll }} / d z$ is the fraction of baryons collapsed to form a protogalaxy per unit redshift, $f_{*}$, the fraction of baryons converted into stars in a single star formation event.

The corresponding heating rate due to DDM can be found as $K_{*}=\chi_{h} \epsilon_{X}$ (Vasiliev \& Shchekinov 2013)

$$
\frac{2 K}{3 k H(z)}=8.9 \times 10^{4}\left(\xi / \xi_{L}\right)\left(\frac{1+z}{10}\right)^{-2 / 3} \text {. }
$$

The dependence of rates Eqs (3), (9), and (10) on the redshift are not very different. So from the global signal it would be difficult to separate between the decaying dark matter, X-ray heating, and magnetic fields.

In other words, the main discriminator between these models is the far larger number of haloes produced owing to additional density perturbations in the presence of primordial magnetic fields.

\section{CONCLUSIONS}

In the paper we studied the impact of primordial magnetic fields on HI absorption from collapsed haloes during the epoch of reionization. We considered haloes in the mass range $10^{6}-10^{8} \mathrm{M}_{\odot}$ and the absorption from these haloes for $r \leq 3 r_{\text {vir }}$. We consider magnetic field strength in the range: $B_{0}=0.3-0.6 \mathrm{nG}$, comparable to the best upper bounds on these fields from cosmological observables (Pandey \& Sethi 2013, 2012; ; Shaw \& Lewis 2012; Kahniashvili et al. 2010, 2013).

The presence of magnetic fields result in two separate effects. The decay of magnetic fields in the collapsing haloes owing to ambipolar diffusion heats the halo which lowers the optical depth of absorption for intermediate masses (Figure 2). Primordial magnetic fields also generate additional density perturbations which result in a sharp increase in the number of haloes (Figure 3). The latter effect dominates the observable signature (Figure 4).

If these haloes become star-forming they could be responsible for early reionization and result in distinct HI fluctuation signature from the epoch of reionization. However, if these haloes remain HI rich then their presence will not significantly impact the HI emission signal from the epoch of reionization as their temperatures are far larger than the CMBR temperature (Figure 1) and the HI emission signal is nearly independent of the spin 
temperature (assuming $T_{s}=T_{k}$ ) in this case. Therefore, the most promising way to observe them would be in absorption.

In this paper we investigate the HI absorption for $z \geq 10$. The clumpiness of HI distribution caused by magnetic fields might leave its signature at lower redshifts also, e.g. in the radiative transfer of radiation from Lyman- $\alpha$ emitting galaxies which are observed up to a redshift $z \simeq 8.5$.
EV acknowledges partial support from the Russian Foundation for Basic Research through the grant 1202-00365 and the "Dynasty" foundation. EV is grateful the Raman Research Institute for hospitality, where this work was begun.

\section{REFERENCES}

Beck, R. 2012, Space Science Review, 166, 215

Brandenburg, A., \& Subramanian, K. 2005, Phys. Rep., 417, 1

Burkert A., 1995, ApJ, 447, L25

Carilli, C. L., Wang, R., van Hoven, M. B., et al. 2007, AJ, 133 2841

Cen R., 1992, ApJ, 78, 341

Chuzhoy L. 2008, ApJL, 679, 65

Cumberbatch D. T., Lattanzi M., Silk J., 2010, PhRvD, 82j3508

Cooray A. \& Furlanetto S.R., 2005, MNRAS, 359, L47

Field G.B., 1958, Proc. IRE, 46, 240

Flower D., 2000, MNRAS, 318, 875

Furlanetto S. R., 2006, MNRAS, 370, 1867

Furlanetto S. R., Oh S. P., Pierpaoli E., 2006, Phys. Rev. D, 74, 103502

Furlanetto, S. R., Oh, S. P., \& Briggs, F. H. 2006, Phys. Rep., 433, 181

Galli D. \& Palla F., 1998, A\&A, 335, 403

Gopal R., Sethi S. K., 2003, JA\&A, 24, 51

Gnedin, N. Y., \& Shaver, P. A. 2004, ApJ, 608, 611

Iliev I.T., Shapiro P.R., Ferrara A., Martel H., 2002, ApJL, 572, 123

Kahniashvili T., Tevzadze A.G., Sethi S.K., Pandey K., Ratra B., Phys. Rev. D, 82, 083005

Kahniashvili T., Maravin Yu., Natarajan A., Battaglia N., Tevzadze A., 2013, ApJ, 770, 47, arXiv:1211.2769

Kim E.-J., Olinto A. V., Rosner R., 1996, ApJ, 468, 28

Kuhlen M., Madau P., Montgomeri R., 2006, ApJL, 637, 1

Lipovka A., Núñez-López R., Avila-Reese V., 2005, MNRAS, 361, 850

Liszt H., 2001, A\&A, 371, 698

Mack, K. J., \& Wyithe, J. S. B. 2012, MNRAS, 425, 2988

Myers Z. \& Nusser A., 2008, MNRAS, 384, 727

Natarajan A. \& Schwarz D. J. 2009, PhRvD, 80d3529

Padmanabhan T., 1993, Structure Formation in the Universe, Cambridge Univ. Press, Cambridge

Pandey, K. L., \& Sethi, S. K. 2013, ApJ, 762, 15

Pandey, K. L., \& Sethi, S. K. 2012, ApJ, 748, 27

Paoletti D. \& Finelli F., 2013, Phys. Lett. B, 726, 45, arXiv:1208.2625

Parker, E. N. 1979, Cosmical Magnetic Field: Their Origin and Their Activity, (Oxford University Press)

Planck Collaboration: Ade P.A.R. et al., 2013, A\&A submitted, arXiv: 1303.5076

Pritchard, J. R., \& Loeb, A. 2010, Phys. Rev. D, 82, 023006

Ratra, B. 1992, ApJL, 391, L1

Ripamonti E., 2007, MNRAS, 376, 709

Ryu D., Schleicher D.R.G., Treumann R.A., Tsagas C.G., Widrow L.M., 2012, Spa. Sci. Rev., 166, 1
Schleicher, D. R. G., Galli, D., Glover, S. C. O., et al. 2009, ApJ, 703, 1096

Schleicher D.R.G., Banerjee R., Klessen R.S., 2009, Phys. Rev. D, 79, 043510

Schleicher, D. R. G., Banerjee, R., \& Klessen, R. S. 2008, Phys. Rev. D, 78, 083005

Sethi S., 2005, MNRAS, 363, 818

Sethi S. K., Subramanian K., 2005, MNRAS, 356, 778

Sethi S.K., Nath B.B., Subramanian K., 2008, MNRAS, 387, 1589

Sethi, S. K., \& Subramanian, K. 2009, JCAP, 11, 21

Sethi, S., Haiman, Z., \& Pandey, K. 2010, ApJ, 721, 615

Shaver P. A., Windhorst R. A., Madau P., \& de Bruyn A. G., 1999, A\&A, 345, 380

Shaw J.R. \& Lewis A., 2012, Phys. Rev. D, 86, 043510, arXiv: 1006.4242

Shchekinov Yu. A. \& Vasiliev E. O., 2004, A\&A, 419, 19 Shchekinov Yu. A. \& Vasiliev E. O., 2007, MNRAS, 379, 1003

Schober, J., Schleicher, D. R. G., \& Klessen, R. S. 2013, A\&A, 560, A87

Spergel D. N., Bean R., Doré O. et al., 2007, ApJS, 170, 377

Stancil P.C., Lepp S., Dalgarno A., 1998, ApJ, 509, 1

Subramanian K., Barrow J. D., 1998, Phys. Rev. D, 58, 083502

Sur, S., Federrath, C., Schleicher, D. R. G., Banerjee, R., \& Klessen, R. S. 2012, MNRAS, 423, 3148

Tashiro H., Sugiyama N., 2006, MNRAS, 368, 965

Thoul A. \& Weinberg D., 1995, ApJ, 442, 480

Tozzi P., Madau P., Meiskin A., Rees M.J., 2000, ApJ, 528, 597

Turner, M. S., Widrow, L. M. 1988, PRD, 37, 2743

Trivedi P., Seshadri T. R., Subramanian K., 2012, PRL, 108, 23

Vasiliev E. O. \& Shchekinov Yu. A., 2006, Astr. Rept., 50, 778

Vasiliev E. O. \& Shchekinov Yu. A., 2012, Astr. Rept., 89, 99

Vasiliev E. O. \& Shchekinov Yu. A., 2013, ApJ, 777, 8

Wasserman I., 1978, ApJ, 224, 337

Widrow, L. M. 2002, Reviews of Modern Physics, 74, 775

Wouthuysen S. 1952, AJ, 57, 31

Xu Y., Ferrara A., Chen X., 2011, MNRAS, 410, 2025

Yamazaki, D. G., Ichiki, K., Kajino, T., Mathews, G. J. 2010, Ad. Astr., 2010, 586590

Yuan Q., Yue B., Bi. X.-J., Chen X., Zhang X., 2010, JCAP, 10 023

Zeldovich, I. B., Ruzmaikin, A. A., Sokolov, D. D. 1983, The Fluid Mechanics of Astrophysics and Geophysics. Volume 3, (New York: Gordon and Breach Science Publishers), 381 p. 